\title{
Editorial
}

\section{The Evolution of Endoscopic Ear Surgery}

Traditionally, tympanoplasty operations has been being performed using microscope but Endoscopic techniques are becoming more popular in otologic surgery. Endoscopic ear surgery (EES) provides several advantages compared to traditional microscopic surgery. Rigid endoscopy allows wide field view of the surgical field, improved resolution with high magnification, and the ability to "look around the corners," enabling direct visualization of the hidden recesses, including the retrotympanum, epitympanum, supratubal recess, protympanum, and hypotympanum ${ }^{1}$

In 1950s fiberoptics were developed and in 1954 Harold Hopkins developed Optical fiber. In 1967 the first investigation of ear with endoscope was done by Mer and colleagues. 1982 Nomura first published a report of middle ear endoscopic exploration through a myringotomy ${ }^{2}$.

Advances in endoscopic instrumentation and video technology allowed Poe and colleagues to describe the use of small diameter endoscopes to explore the middle ear through a strategically placed myringotomy in the early $1990 \mathrm{~s}^{3}$. Later that decade, MuaazTarabichi described middle ear surgery performed exclusively via endoscopic visualization ${ }^{4-7}$.

In the 2000s The International Working Group on Endoscopic Ear Surgery (WGEESI) formed as a consortium of otologists interested in endoscopic ear surgery.

In Bangladesh Endoscopic Ear Surgery was started around 2005. Initially Transcanal Myringoplasty were done. Now other middle ear surgeries like Stapedotomy,
Cholesteatoma surgeries are done with good results. To improve the skills and knowledge, Endoscopic Cadaveric Temporal bone Dissection courses are organized for graduate students, residents \& junior ENT specialists regularly.

Modern high-definition video systems now provides superior image quality that has made endoscopic middle ear surgery a viable alternative to the microscopic techniques in several centers in the United States and abroad, but there has been slow adoption of EES due to good outcomes with traditional techniques, lack of EES exposure during residency and fellowship training, limited instrumentation, and concerns about one handed surgery and poor depth perception. However, Endoscope learning curve of EES is higher than microscope.

Traditional microscopic approaches provide the benefits of two-handed dissection and depth perception offered by binocular vision (despite the possible need for increased drilling and tissue retraction) and greater proficiency by most surgeons associated with adequate exposure during training. In contrast, proponents of EES suggest several distinct advantages over the microscope, such as improved visualization and access to the hidden recesses of the middle ear, avoidance of a postauricular incision in many cases, and less bony drilling. In ideal form, this minimally invasive approach holds the promise of decreased operative time, less postoperative morbidity, and diminished need for mastoidectomy surgery.

The most widely studied application of EES is for management of cholesteatoma. This 
indication exploits the biggest advantage of the endoscope: the ability to look around corners when using a transcanal approach without depending on lineof-sight surgery needed when using the binocular microscope ${ }^{1}$. We seek to quantify disease control through a comparison of residual/ recurrent disease. Additionally, we seek to further highlight other applications of EES, including myringoplasty, tympanoplasty, ossiculoplasty, and relevant hearing outcomes, as well as described outcomes with other otologic procedures, such as cochlear implantation, stapedectomy, and approaches to the lateral skull base.

The spectrum of EES is growing in scope and frequency of use, while being applied to a broad range of middle ear and skull base pathologies. Current data suggest that EES provides equivalent short-term and long-term control of chronic middle ear disease (cholesteatoma) compared with microscopic approaches, while offering the potentially decreased morbidity of a minimally invasive approach. Outcomes following tympanoplasty, ossiculoplasty, and stapedectomy are also promising in terms of delivering equivalent hearing outcomes via an endoscopic approach. Despite a rapidly growing collection of retrospective analyses and case series, there remains a distinct lack of large prospective studies to better evaluate the short-term and long-term outcomes of EES in relation to the gold standard of surgical microscopy, as well as patient-centered outcome studies that can quantify benefit of minimally invasive surgery. Additional retrospective and prospective studies are needed to further classify the efficacy and presumed reduced morbidity of endoscopic middle ear surgery. The role of the endoscope beyond tympanoplasty/mastoidectomy surgery is still evolving. A number of smaller studies suggest that the endoscope can be a valuable adjunct during vestibular schwannoma resection and approaches to the petrous apex, and anatomic studies of transcanal endoscopic corridors to the skull base suggest that endoscopy may play an enlarging role in lateral skull base surgery. Additional studies and refinement of operative techniques will be critical to these applications of EES in the future ${ }^{1-2}$

\section{Prof. Dr. Md. MonjurulAlam}

Professor, Dept. of Otolaryngology - Head and Neck Surgery, Bangabandhu Sheikh Mujib Medical University Phone: +8801819227717 Email: entdralam@gmail.com

\section{References}

1. Marchioni D, Villari D, Mattioli F, Ciutelli M. Piccinini A. Presutti L, Otolaryngo IClin N Am 46 (2013) 201-209.

2. Kiringoda R. Kozin E, Lee D. OtolaryngolClin N Am 49 (2016) 12711290

3. MerSB,DerbyshireAJ,BrushenkoA, et al. Fiberopticendotoscopesforexamining the middle ear. Arch Otolaryngol 1967;85(4):387-93.

4. Poe DS, Rebeiz EE, Pankratov MM, et al. Transtympanic endoscopy of the middle ear. Laryngoscope 1992;102(9): 993-6.

5. Tarabichi M. Endoscopic management of acquired cholesteatoma. AmJOtol 1997;18(5):544-9.

6. Tarabichi M. Endoscopic middle ear surgery. Ann OtolRhinolLaryngoll999; 108(1):39-46.

7. Tarabichi M. Endoscopic management of cholesteatoma: long-term results. Otolaryngol Head Neck SurglOOO; 122(6):874-81. 